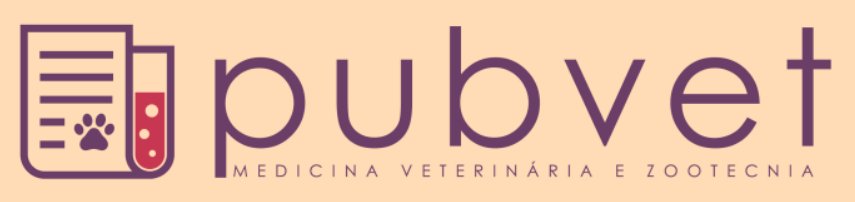

https://doi.org/10.22256/pubvet.v12n3a44.1-4

\title{
Corticoterapia em um cão com choque séptico não responsivo a vasopressores: Relato de caso
}

\author{
Breno Curty Barbosa ${ }^{\ominus} 1^{*}$, Fernanda dos Santos Alves ${ }^{\ominus 1}$, Nathália das Graças Dorneles Coelho ${ }^{\ominus}$, \\ Paula Costa de Oliveira Pinto ${ }^{2}$, Suzane Lilian Beier ${ }^{\bullet}$, Patrícia Maria Coletto Freitas ${ }^{\bullet} 3$
}

${ }^{I}$ Doutorando em Ciência Animal, Universidade Federal de Minas Gerais, Departamento de Clínica e Cirurgia Veterinária. Belo Horizonte-MG, Brasil. ${ }^{2}$ Mestrando em Ciência Animal da Universidade Federal de Minas Gerais, Departamento de Clínica e Cirurgia Veterinárias. Belo Horizonte - MG, Brasil. ${ }^{3}$ Docente da Universidade Federal de Minas Gerais, Departamento de Clínica e Cirurgia Veterinárias. Belo Horizonte - MG, Brasil.

*Autor para correspondência, E-mail: brenocurty@hotmail.com

RESUMO. O choque séptico, consequência de um quadro de sepse grave com hipotensão não responsiva a reposição volêmica, apresenta-se de forma bastante comum em medicina veterinária, e sua ocorrência pode vir consorciada com deficiência absoluta ou relativa de hormônios corticosteroides, sendo assim necessária a reposição destes. Um cão foi atendido no Hospital Veterinário em choque séptico, iniciou-se a terapia com solução cristaloide, seguida de vasopressores, não havendo melhora no quadro do animal. Vinte e quatro horas após o início deste tratamento, optou-se pela administração de hidrocortisona, observando em seguida melhora gradual nos parâmetros hemodinâmicos. Após 3 dias de uso da hidrocortisona, observou-se uma melhora significativa no quadro clínico do animal. Assim, conclui-se que o uso de corticoide pode ser necessário em quadro de choque séptico não responsivo a vasopressores.

Palavras chave: canino, corticosteroides, insuficiência adrenocortical

\section{Cortico-therapy in a dog with septic shock not responsive to vasopressors: $A$ case report}

\begin{abstract}
Septic shock, a consequence of severe sepsis with hypotension that is not responsive to volume replacement, is quite common in veterinary medicine, and its occurrence may be consorted with absolute or relative deficiency of corticosteroid hormones, thus requiring replacement of these. A dog was treated at the Veterinary Hospital in septic shock, therapy was started with crystalloid solution, followed by vasopressors, and there was no improvement in the animal's picture. Twenty-four hours after the start of this treatment, hydrocortisone was administered, and then gradual improvement in hemodynamic parameters was observed. After 3 days of hydrocortisone use, a significant improvement in the clinical picture of the animal was observed. Thus, it is concluded that the use of corticosteroids may be necessary in patients with septic shock who are not responsive to the vasopressors.
\end{abstract}

Keywords: canine, corticosteroids, adrenocortical insufficiency

\section{Corticoterapia en un perro con choque séptico no respondiente a vasopresores: Reporte de un caso}

RESUMEN: El choque séptico, consecuencia de un cuadro de sepsis grave con hipotensión no responsiva a la reposición volémica, se presenta comúnmente en medicina veterinaria, y su ocurrencia puede venir consorciada con deficiencia absoluta o relativa de 
hormonas corticosteroides, siendo necesaria la reposición de estos. Un perro fue atendido en el Hospital Veterinario en shock séptico, se inició la terapia con solución cristaloide, seguida de vasopresores, no habiendo mejoría en el cuadro del animal. Veinticuatro horas después del inicio de este tratamiento, se optó por la administración de hidrocortisona, observando en seguida una mejora gradual en los parámetros hemodinámicos. Después de 3 días del uso de hidrocortisona, se observó una mejora significativa en el cuadro clínico del animal. Así, se concluye que el uso de corticoide puede ser necesario en cuadro de choque séptico no respondiente a vasopresores.

Palabras clave: canino, corticosteroides, insuficiencia adrenocortical

\section{Introdução}

A sepse é definida como a resposta inflamatória sistêmica do hospedeiro a uma infecção, seja ela por vírus, fungos ou bactérias. $\mathrm{Na}$ sepse grave observa-se um quadro de sepse associado com uma disfunção orgânica e alterações de perfusão, podendo desenvolver insuficiência adrenocortical (Theobaldo, 2012). O choque séptico é definido pela presença de hipotensão arterial refratária à expansão de volume e que, portanto, necessita do uso de vasopressores e inotrópicos para sua correção (Lopes et al., 2010, Rabelo, 2013). Todavia, muitas controvérsias surgem em sua terapêutica principalmente quanto ao uso dos glicocorticoides. Dentre os questionamentos estão como e em qual momento, qual dose a ser utilizada e principalmente sobre qual corticoide empregado, são comuns nos setores de emergência e terapia intensiva, seja na medicina humana ou veterinária (Sprung et al., 2008). Estudos demonstraram que após administração de baixas doses de corticoides em humanos com choque séptico, verificou-se uma redução da produção de citocinas próinflamatórias, inativação de células endoteliais e neutrófilos, cursando com não geração da fase aguda da inflamação (Annane et al., 2009).

Objetivou-se com este relato descrever o uso de corticoide em um cão com choque séptico não responsivo a vasopressores.

\section{Relato de caso}

Foi admitido no setor de emergência do Hospital Veterinário da Escola de Veterinária da Universidade Federal de Minas Gerais, um cão macho, com oito meses de idade, sem histórico de vacinação e vermifugação, pesando $12,5 \mathrm{~kg}$, da raça Rottweiller cursando com apatia, intensa sialorreia, frequência cardíaca (FC) de 140 batimentos por minuto (bpm), frequência respiratória (FR) de 26 movimentos por minuto (mpm), temperatura retal de $39,8^{\circ} \mathrm{C}$, tempo de preenchimento capilar (TPC) de 3 segundos, glicemia de $130 \mathrm{mg} / \mathrm{dL}$ com pressão arterial sistólica (PAS) de $40 \mathrm{mmHg}$, pulso periférico filiforme e tugor cutâneo elevado. Deu-se início a restituição volêmica com três provas de carga de $20 \mathrm{~mL} / \mathrm{kg}$, com duração de 15 minutos cada uma, verificando-se após a conduta uma PAS de 66 $\mathrm{mmHg}$. Iniciou-se o uso de norepinefrina sob infusão contínua em uma taxa de $0,6 \mathrm{mcg} / \mathrm{kg} / \mathrm{min}$ e observou-se uma discreta elevação na PAS (78 $\mathrm{mmHg}$ ), sendo associado infusão contínua de dobutamina na taxa de $5 \mathrm{mcg} / \mathrm{kg} / \mathrm{min}$ que sustentou a PAS entre $85-90 \mathrm{mmHg}$. Diante do histórico e quadro clínico apresentado pelo paciente, uma das hipóteses diagnósticas era a Síndrome da Gastroenterite Hemorrágica. Por isso foi realizado o teste rápido para parvovirose canina e forma de ELISA, com resultado positivo.

No segundo dia houve piora do estado geral do animal, com desenvolvimento de prostração intensa, decúbito lateral, FC de 164 bpm, PAS de $40 \mathrm{mmHg}$, FR $60 \mathrm{mpm}$. Foram realizadas mais duas provas de carga, além de elevar-se a taxa de infusão da norepinefrina para $0,8 \mathrm{mcg} / \mathrm{kg} / \mathrm{min}$ e a dobutamina para $15 \mathrm{mcg} / \mathrm{kg} / \mathrm{min}$. Após 1 hora da nova terapia, observou-se PAS de $45 \mathrm{mmHg}$. Optou-se pela administração de hidrocortisona na dose de $0,5 \mathrm{mg} / \mathrm{kg}$ e após 8 horas da aplicação verificou-se uma elevação da PAS para $70 \mathrm{mmHg}$. A hidrocortisona foi mantida a cada 6 horas, por 3 dias consecutivos; seguidos de administrações a cada 12 horas por mais 2 dias, por via intravenosa.

Após 24 horas da primeira administração da hidrocortisona verificou-se uma PAS de 90 $\mathrm{mmHg}, \mathrm{FC}$ de $154 \mathrm{bpm}$, FR $60 \mathrm{mpm}, \mathrm{T}^{\circ} \mathrm{C}$ retal de 39,1. As infusões de norepinefrina e dobutamina continuaram a serem administradas, porém a norepinefrina foi reduzida para um valor de 0,1 $\mathrm{mcg} / \mathrm{kg} / \mathrm{min}$ e dobutamina $5 \mathrm{mcg} / \mathrm{kg} / \mathrm{min}$. Ao entrar no quarto dia de internação e no terceiro dia de hidrocortisona observou-se uma melhora significativa do quadro, sustentando uma PAS de $120 \mathrm{mmHg}$, com retirada os fármacos vasopressores e inotrópicos, além de suspensão da 
hidrocortisona no quinto dia. Com a evolução clínica satisfatória no decorrer dos dias, com os parâmetros fisiológicos dentro dos valores da normalidade para a espécie, e melhora do quadro clínico, o paciente foi transferido para o canil do setor da clínica médica, recebendo alta em seguida. Ressalva-se que o paciente recebeu todo o tratamento suporte desde a sua admissão como antibioticoterapia em sua primeira hora de internação (amoxicilina com ácido clavulânico 22 $\mathrm{mg} / \mathrm{kg}$ a cada 12 horas, IV; metronidazol $15 \mathrm{mg} / \mathrm{kg}$ a cada 12 horas, IV). O cálculo de fluidoterapia foi baseado no grau de desidratação, necessidade diária (manutenção), perdas insensíveis e por vômito e diarreia. Para controle dos vômitos, optou-se por via subcutânea, maropitant $(1 \mathrm{mg} / \mathrm{kg}$ a cada 24 horas) e para controle álgico e conforto do paciente a adminstração da dipirona $(25 \mathrm{mg} / \mathrm{kg}$ a cada 8 horas).

\section{Discussão}

No presente relato o animal apresentava choque séptico, ou seja, possuía alteração na frequência cardíaca e respiratória, temperatura corpórea, estado mental e hipotensão refratária à expansão de volume, conforme classificação descrita por Hauptman et al. (1997), Lopes et al. (2010) e Rabelo (2013). Assim, seguindo a recomendação de Lopes et al. (2010) iniciou-se a reposição volêmica com uso de cristaloide porém sem sucesso, pois não conseguiu-se a estabilização da pressão arterial sistólica (PAS). Contudo, iniciou-se a infusão contínua de norepinefrina e dobutamina, conforme descrito no protocolo da Campanha de Sobrevivência à Sepse (Surviving Sepsis Campaign), devido esta ser o vasopressor de primeira escolha para pacientes em choque séptico, pois promove ação vasoconstritora sistêmica pela ativação de receptores $\alpha$-adrenérgicos (Guinot et al., 2015). A associação do inotrópico positivo, no caso dobutamina, foi realizada neste paciente com o objetivo de elevação do débito cardíaco. Os fármacos vasopressores como norepinefrina provocam uma elevação do tônus vascular e pressórica, porém consorciam-se com queda significativa no débito cardíaco e fluxo sanguíneo dos capilares, principalmente cutâneos, esplênico e renal (Silverstein \& Beer, 2015).

Mesmo com a infusão de dois vasopressores, em altas doses, não se observou uma elevação na pressão sanguínea sistólica, assim suspeitou-se de que o animal encontrava-se em um quadro denominado choque refratário, em que ocorre uma insuficiência das glândulas adrenais (absoluta ou relativa). As principais causas dessas insuficiências, de acordo com Annane \& Cavaillon (2003) estão relacionadas à inflamação sistêmica, que reduz a afinidade dos receptores $\alpha$ e $\beta$-adrenérgicos nos tecidos, levando a uma baixa resposta aos mineralocorticoides, principalmente devido à interleucina tipo 2 , que se encontra em valores elevados nesses quadros.

Como se suspeitou que o cão estivesse em choque refratário optou-se acrescentar na terapia a hidrocortisona em baixa dose, pois segundo Goodman \& Sprung (2002), o uso dos corticoides, em destaque hidrocortisona, nestes casos ajudaria a reativar esses receptores adrenérgicos, melhorando os quadros hemodinâmico e sistêmico. Além disso, de acordo com Sprung et al. (2008) e Lima \& Franco (2010), baixas doses de hidrocortisona são indicadas em hipotensão refratária a ressuscitação volêmica. O emprego de vasoativos e inotrópicos após uma hora da sua administração em doses altas, como ocorreu com o paciente supracitado, ressaltando uma redução da sua dosagem após estabilização do quadro sistêmico, com objetivo de sensibilizar os receptores vasopressóricos.

A dose empregada no paciente foi de $0,5 \mathrm{mg} / \mathrm{kg}$ a cada 6 horas durante três dias, sendo espaçada para cada 12 horas durante mais 2 dias. Essa posologia foi baseada no estudo retrospectivo de Creedon (2015), que também sugere o emprego da administração da hidrocortisona em forma de infusão contínua na taxa de $0,08 \mathrm{mg} / \mathrm{kg} / \mathrm{h}$ como terapia optativa.

\section{Conclusão}

O uso de corticoides pode ser necessário em quadro de choque séptico não responsivo a vasopressores. Contudo, seu uso ainda envolve mistério, associado a poucos relatos em medicina veterinária. Novos estudos se fazem necessários para avaliar o momento exato da administração do agente.

\section{Referências Bibliográficas}

Annane, D., Bellissant, E., Bollaert, P.-E., Briegel, J., Confalonieri, M., De Gaudio, R., Keh, D., Kupfer, Y., Oppert, M. \& Meduri, G. U. 2009. Corticosteroids in the treatment of severe sepsis and septic shock in adults: a systematic review. Jama, 301, 2362-2375. 
Annane, D. \& Cavaillon, J.-M. 2003. Corticosteroids in sepsis: from bench to bedside? Shock, 20, 197-207.

Creedon, J. M. B. 2015. Controversies surrounding critical illness-related corticosteroid insufficiency in animals. Journal of Veterinary Emergency and Critical Care, 25, 107-112.

Goodman, S. \& Sprung, C. L. 2002. The International Sepsis Forum's controversies in sepsis: corticosteroids should be used to treat septic shock. Critical Care, 6, 381-383.

Guinot, P.-G., Bernard, E., Levrard, M., Dupont, H. \& Lorne, E. 2015. Dynamic arterial elastance predicts mean arterial pressure decrease associated with decreasing norepinephrine dosage in septic shock. Critical Care, 19, 1-7.

Hauptman, J. G., Walshaw, R. \& Olivier, N. B. 1997. Evaluation of the sensitivity and specificity of diagnostic criteria for sepsis in dogs. Veterinary Surgery, 26, 393-397.

Lima, A. F. K. \& Franco, R. P. 2010. Síndrome da resposta inflamatória sistêmica (SRIS), um desafio diagnóstico. Acta Veterinaria Brasilica, 4, 123-131.

Lopes, R. D., Silva, A. M. B. \& Bonillha, A. M. M. 2010. Sepse grave e choque séptico: aspectos clínicos. In: Guimarães, H. P., Lopes, R. D. \& Lopes, A. C. (eds.) Tratado de medicina de urgência, emergência, prontosocorro e UTI. Editora Atheneu, São Paulo, São Paulo.

Rabelo, R. C. 2013. Sepse, sepse grave e choque séptico. In: Rabelo, R. (ed.) Emergências de pequenos animais - Condutas clínicas e cirúrgicas no paciente grave. Elsevier, Rio de Janeiro.

Silverstein, D. C. \& Beer, K. A. S. 2015. Controversies regarding choice of vasopressor therapy for management of septic shock in animals. Journal of Veterinary Emergency and Critical Care, 25, 48-54.

Sprung, C. L., Annane, D., Keh, D., Moreno, R., Singer, M., Freivogel, K., Weiss, Y. G., Benbenishty, J., Kalenka, A. \& Forst, H. 2008. Hydrocortisone therapy for patients with septic shock. New England Journal of Medicine, 358, 111-124.

Theobaldo, M. C. 2012. Efeitos da solução salina hipertônica na resposta inflamatória na sepse. Universidade de São Paulo, São Paulo.

Article History:

Received 6 November 2017

Accepted 14 December 2017

Available online 8 February 2018

License information: This is an open-access article distributed under the terms of the

Creative Commons Attribution License 4.0, which permits unrestricted use, distribution, and reproduction in any medium, provided the original work is properly cited. 